\title{
A Novel OGR1 (GPR68) Inhibitor Attenuates Inflammation in Murine Models of Colitis
}

\author{
Cheryl de Vallière $^{\mathrm{a}} \quad$ Katharina Bäbler $^{\mathrm{a}} \quad$ Philipp Busenhart $^{\mathrm{a}}$ \\ Marlene Schwarzfischer ${ }^{a} \quad$ Chiaki Maeyashiki $^{a} \quad$ Cordelia Schuler $^{a} \quad$ Kirstin $_{\text {Atrott }}{ }^{a}$ \\ Silvia Lang ${ }^{a}$ Marianne R. Spalinger ${ }^{a}$ Michael Scharla, b Pedro A. Ruiz-Castro ${ }^{a}$ \\ Martin Hausmann ${ }^{a}$ Gerhard Rogler ${ }^{a, b}$ \\ ${ }^{\text {aD }}$ epartment of Gastroenterology and Hepatology, University Hospital Zurich, (USZ), University of Zurich, Zurich, \\ Switzerland; bZurich Center for Integrative Human Physiology, Zurich, Switzerland
}

\section{Keywords}

Ovarian cancer G protein-coupled receptor 1 antagonist . $\mathrm{pH}$-sensing $\mathrm{G}$ protein-coupled receptor · Inflammatory bowel disease

\begin{abstract}
Background and Aims: Local extracellular acidification is associated with several conditions, such as ischemia, cancer, metabolic disease, respiratory diseases, and inflammatory bowel disease (IBD). Several recent studies reported a link between IBD and a family of $\mathrm{pH}$-sensing $\mathrm{G}$ protein-coupled receptors. Our previous studies point to an essential role for OGR1 (GPR68) in the modulation of intestinal inflammation and fibrosis. In the current study, we evaluated the effects of a novel OGR1 inhibitor in murine models of colitis. Methods: The effects of a novel small-molecule OGR1 inhibitor were assessed in the acute and chronic dextran sulfate sodium (DSS) murine models of colitis. Macroscopic disease indicators of intestinal inflammation were evaluated, and epithelial damage and immune cell infiltration and proliferation were assessed by immunohistochemistry. Results: The OGR1 inhibitor ameliorated clinical parameters in acute and chron-
\end{abstract}

ic DSS-induced colitis. In mice treated with the OGR1 inhibitor, endoscopy showed no thickening and normal vascularity, while fibrin was not detected. Histopathological findings revealed a decrease in severity of colonic inflammation in the OGR1 inhibitor group when compared to vehicle-DSS controls. In OGR1 inhibitor-treated mice, staining for the macrophage marker F4/80 and cellular proliferation marker Ki-67 revealed a reduction of infiltrating macrophages and slightly enhanced cell proliferation, respectively. This was accompanied by a reduction in pro-inflammatory cytokines, TNF and IL-6, and the fibrosis marker TGF- $\beta 1$. Conclusion: This is the first report providing evidence that a pharmacological inhibition of OGR1 has a therapeutic effect in murine colitis models. Our data suggest that targeting proton-sensing OGR1 using specific small-molecule inhibitors may be a novel therapeutic approach for the treatment of IBD.

(c) 2021 The Author(s).

Published by S. Karger AG, Basel

Cheryl de Vallière and Katharina Bäbler contributed equally to this work and share first authorship. Pedro A. Ruiz-Castro, Martin Hausmann, and Gerhard Rogler share senior authorship.

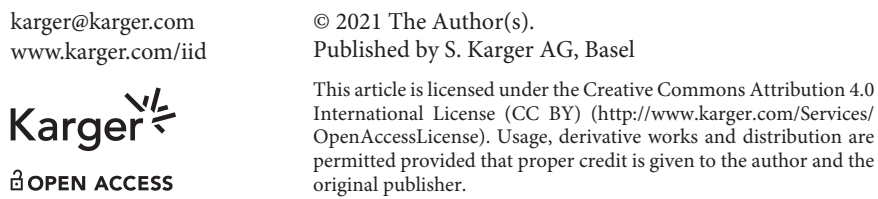

karger@karger.com www.karger.com/iid

Karger $\stackrel{\text { ' }}{=}$

BOPEN ACCESS

(C) 2021 The Author(s)

Published by S. Karger AG, Basel

This article is licensed under the Creative Commons Attribution 4.0 International License (CC BY) (http://www.karger.com/Services/ OpenAccessLicense). Usage, derivative works and distribution are permitted provided that proper credit is given to the author and the original publisher.

Correspondence to:

Cheryl de Vallière, cheryl.devalliere@usz.ch

Martin Hausmann, martin.hausmann@usz.ch

Gerhard Rogler, gerhard.rogler@usz.ch 


\section{Introduction}

Acidic tissue microenvironments exist in many types of inflammatory disorders, including inflammatory bowel disease (IBD) [1-3]. To maintain $\mathrm{pH}$ homeostasis, it is necessary that cells sense changes in extracellular $\mathrm{pH}$ and respond accordingly. A family of $\mathrm{pH}$-sensing $\mathrm{G}$ proteincoupled receptors (GPCRs) has been identified to play an important role in physiological $\mathrm{pH}$ homeostasis [4]. This family of transmembrane receptors include ovarian cancer $\mathrm{G}$ protein-coupled receptor 1 (OGR1 also known as GPR68), G protein-coupled receptor 4 (GPR4), and Tcell death-associated gene 8 (TDAG8 also known as GPR65). These receptors are activated by extracellular protons and in turn stimulate second messenger signaling pathways [4-6], which lead to gene transcription and the modification of various cellular functions. OGR1, which is inactive at $\mathrm{pH} 7.8$ but fully active at $\mathrm{pH} 6.8$, couples predominantly with $\mathrm{G}_{\mathrm{q} / 11}$, stimulating inositol trisphosphate formation and intracellular $\mathrm{Ca}^{2+}$ release [4]. However, OGR1-mediated Gs/cyclic adenosine monophosphate formation and $\mathrm{G}_{\alpha 12 / 13}$ RhoA activity have been reported in several cell types [7-10].

IBD affects approximately one in 150 people in the industrialized world; however, in developing countries, the incidence and prevalence of IBD continues to increase [11]. To date, there is no known cure for IBD, and currently the focus is on symptomatic management of the disease. Current treatments include therapeutic agents that reduce inflammation, such as corticosteroids, immunosuppressive drugs, and anti-TNF monoclonal antibodies, as well as surgical intervention. As the global public health burden of IBD is substantial, there is considerable incentive to develop new treatments for IBD [12].

Although the two major forms of IBD, Crohn's disease and ulcerative colitis, are two distinct diseases, they share many clinical and pathological features. Both diseases are characterized by acute flare ups and symptom-free phases, in which patients suffer from abdominal pain, diarrhea, weight loss, rectal bleeding, fatigue, and other extraintestinal symptoms $[13,14]$. Pathological processes include breakdown of the mucosal epithelial barrier, chronic inflammation, and tissue remodeling with fibrosis $[15,16]$. Extensive immune cell infiltration, resulting in loss of intestinal architecture and tissue destruction, is commonly observed in both Crohn's disease and ulcerative colitis [17]. Gut-wall inflammation, typically present in IBD, is associated with extracellular tissue acidification $[2,18,19]$. During inflammation, the increase in local proton concentration arises from infiltration and
Table 1. Data on the OGR1 inhibitor (GPR68-I)

\begin{tabular}{lr}
\hline Characteristics of compound GPR68-I \\
\hline Mol weight, g/mol & 375.4 \\
Solubility in water, $\mu \mathrm{M}$ & 120 \\
Cytotoxicity, $\mu \mathrm{M}$ & 100 \\
IC ${ }_{50}^{*}$ & 12 \\
$\quad$ Human, nM & 89 \\
$\quad$ Mouse, nM & 17 \\
Mouse PK & 91.2 \\
$\quad$ Bioavailability PO, \% & 80.5 \\
$\quad$ AUC PO, ng h/mL & \\
$\quad$ Clearance IV, mL/min/kg & \\
\hline & $*$ \\
& Vhinese hamster ovary-K1 cells overexpressing mouse or human \\
OGR1, upon exposure to acidic pH. OGR1, ovarian cancer G
\end{tabular}

activation of immune cells. This leads to increased energy and oxygen demand, as well as enhanced glucose consumption via glycolysis, which results in an increase in lactic acid formation [19-23].

Recently, we and other groups reported a link between IBD and this family of $\mathrm{pH}$-sensing GPCRs [9, 24-30]. We observed that the genetic ablation of OGR1 in the interleukin (IL) $-10^{-/-}$murine model of spontaneous colitis ameliorated intestinal inflammation [24]. In the present study, we report that an OGR1 antagonist, GPR68-I, reduced the severity of intestinal inflammation in acute and chronic murine dextran sulfate sodium (DSS)-induced colitis models. Our data suggest that targeting $\mathrm{pH}$-sensing receptor OGR1 with small-molecule inhibitors may be a novel therapeutic approach for the treatment of IBD.

\section{Materials and Methods}

\section{Reagents}

All chemicals were obtained from Sigma-Aldrich (St. Louis, MO, USA), unless otherwise stated. All cell culture reagents were obtained from Thermo Fisher Scientific (Reinach, Switzerland), unless otherwise specified. The OGR1 small-molecule inhibitor (GPR68-I; molecular weight, $375.4 \mathrm{~g} / \mathrm{mol} ; \mathrm{IC}_{50}$ in recombinant Chinese Hamster Ovary-K1 cells overexpressing mouse OGR1, 89 nM; cytotoxicity, $100 \mu \mathrm{M}$; Table 1) was kindly provided by Takeda Pharmaceuticals, San Diego, CA, USA.

\section{Animal Models}

All animal experiments were performed according to Swiss animal welfare laws and were approved by the Veterinary Office of the Canton Zurich, Switzerland. The generation, breeding, and ge- 

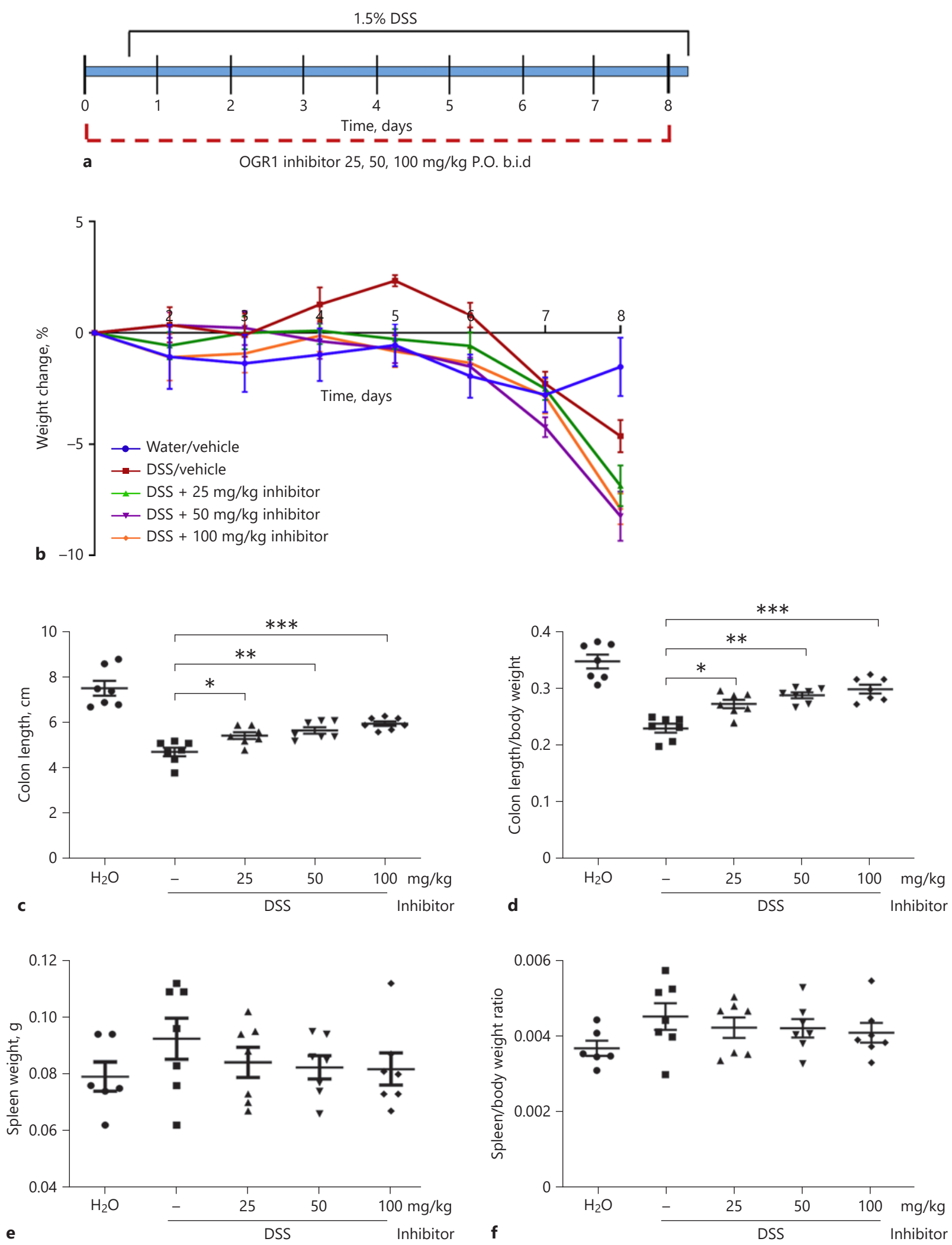

1

(For legend see next page.) 
notyping of OGR1 deficient $\left(\mathrm{Ogr} 1^{-/-}\right) \mathrm{C} 57 \mathrm{BL} / 6$ mice, initially obtained from Deltagen, Inc., San Mateo, CA, USA, have been previously described [24, 31]. Littermates were used in all experiments. Mice were cohoused to minimize any potential effects due to different microbiota. Mice received standard laboratory mouse food and water ad libitum and were housed under specific pathogenfree conditions in a regular day-night cycle in individually ventilated cages with standard bedding and cage enrichment.

\section{Induction of Dextran Sulfate Sodium (DSS) Colitis}

Acute experimental colitis was induced in C57BL/6 wild-type (WT) mice by the addition of $1.5 \%$ (wt/vol) DSS (Mol wt 36-50 $\mathrm{kDa}$; MP Biomedicals, Santa Ana, CA, USA) in the drinking water ad libitum for 7 consecutive days. In the chronic DSS model, colitis was induced in C57BL/6 WT and $O g r 1^{-/-}$mice with 3 cycles of $2 \%$ DSS in drinking water for 7 days, followed by 10 days of DSSfree drinking water, ad libitum. Both female and male mice were used for these experiments, which weighed approximately $20 \mathrm{~g}$ and were 10-16 weeks old when the experiment was started. Mouse body weight and clinical phenotype were assessed daily or 4-6 times per week for the acute and the chronic DSS model, respectively.

For inhibitor application and initial testing dosage, advice from Takeda was followed. For the acute colitis model, the OGR1 inhibitor was administered by oral gavage in a vehicle solution (methylcellulose $0.5 \%$ ), twice daily (b.i.d.) at the 3 recommended test doses: 20, 50, or $100 \mathrm{mg} / \mathrm{kg}$ per day (interval of $\sim 12 \mathrm{~h}$ ). In both acute and chronic models, the water and DSS control groups were gavaged with vehicle solution. In the chronic DSS colitis model, the OGR1 inhibitor was administered in the water recovery period by oral gavage twice daily (b.i.d.) at $50 \mathrm{mg} / \mathrm{kg}$ day (optimal dosage obtained from dosage testing in the acute model). In the acute and chronic models, mice were sacrificed, and inflammatory parameters were evaluated on day 8 and day 55 , respectively.

\section{Evaluation of Inflammation in Murine Colitis}

Mucosal damage was assessed by the Murine Endoscopic Index of Colitis Severity (MEICS), as previously described [32, 33]. Animals were anesthetized intraperitoneally with $90-120 \mathrm{mg}$ of ketamine (Narketan 10\%; Vétoquinol AG, Bern, Switzerland) and 8 mg of xylazine (Rompun 2\%; Bayer, Zurich, Switzerland) per kg body weight and examined by colonoscopy (Karl Storz Tele Pack Pal 20043020; Karl Storz Endoskope, Tuttlingen, Germany). Inflammatory parameters of colitis were evaluated as previously described [28].

\section{Immunohistochemistry}

Immunohistochemistry was performed as previously described [28]. In brief, the last centimeter of the distal part of the colon and $3 \mathrm{~cm}$ of the proximal part of the colon were removed and fixed in $4 \%$ formalin for $24 \mathrm{~h}$. Hematoxylin and eosin (H\&E)-stained sec-

Fig. 1. OGR1 inhibitor reduces clinical severity in acute DSS-induced colitis. Mice were administered with the OGR1 inhibitor twice a day (b.i.d) during the experimental time course (a). Body weight changes (b) were monitored daily and expressed as relative change of body weight in \% relative to day 0 . Mouse colon length (c, d) and splenic enlargement $(\mathbf{e}, \mathbf{f})$ were also assessed upon tissue collection. Statistical analysis was performed using the $t$ test com- tions of the paraffin-embedded tissue were used for histological analysis. Scoring was performed by 2 independent, blinded investigators, as described previously [33]. Immunostaining for F4/80, Ki-67, and CD3 was performed on Leica Bond Max instruments using Refine HRP-Kits (Leica DS9800) and buffer solutions from Leica Microsystems Newcastle according to the manufacturer's guidelines. Paraffin slides were dewaxed, pretreated, and incubated with primary antibody (monoclonal rat anti-mouse F4/80, clone BM8, Cat No. T-2028; BMA Biomedicals, Augst, Switzerland; Ki-67/MKI67 monoclonal rabbit anti-human clone SP6, Cat No. NB600-1252; Novus Biological Inc., Centennial, CO, USA; CD3, rabbit anti-human, clone SP7, monoclonal. Cat No. RMAB005; Diagnostic BioSystems, Pleasanton, CA, USA), at $1: 150,1: 50$, and 1:100 dilutions, respectively, and incubated for 60 $\min$ at $37^{\circ} \mathrm{C}$. For quantification, 12 random pictures were taken from terminal colon sections of each mouse. The resulting pictures (all representing an equal area of the tissue) were quantified by using ImageJ Analysis Software (National Institutes of Health) using the software's color threshold tool, which calculates the area of positive staining. The resulting value was normalized to the values obtained following quantification of nucleus staining and represents the positively stained area normalized to cell numbers present in the given area.

\section{RNA Extraction and Real-Time Quantitative PCR}

Distal colon samples were disrupted using the GentleMACS Dissociator (Miltenyi Biotech, Gladbach, Germany). Total RNA was isolated using the RNeasy Mini Kit (Qiagen, Hombrechtikon, Switzerland) or the Maxwell 16 Total RNA Purification Kit (Promega, Dübendorf, Switzerland), with DNase treatment, following the manufacturer's recommendations. The High-Capacity cDNA Reverse Transcription Kit (Applied Biosystems, Waltham, MA, USA; Thermo Fisher Scientific) for reverse transcription was used following the manufacturer's instructions. qPCR was performed on a Fast HT7900 Real-Time PCR system (Applied Biosystems) using the following PCR program: $20 \mathrm{~s}$ at $95^{\circ} \mathrm{C}$, followed by 40 cycles of $95^{\circ} \mathrm{C}$ for 3 and $60^{\circ} \mathrm{C}$ for $30 \mathrm{~s}$ using the TaqMan FAST Universal Mastermix. Relative mRNA expression was determined by the comparative $\Delta \Delta C$ T method using the reference gene $\beta$-actin. All gene expression assays were obtained from Applied Biosystems: TNF, Mm99999068_m1; IL-6, Mm00446190_m1; TGF- $\beta 1$, Mm01178820_m1; and $\beta$-actin, Mm4352341E.

\section{Statistical Analysis}

Statistical analyses were performed using GraphPad Prism (Version 8.0; GraphPad Software, La Jolla, CA, USA). The unpaired $t$ test was used to compare differences between 2 groups. When comparing 3 or more groups, one-way ANOVA was performed followed by the post hoc Tukey's test. Throughout this article, asterisks denote significant differences at ${ }^{*} p<0.05,{ }^{* *} p<$ 0.01 , and ${ }^{* * *} p<0.001$.

paring vehicle- and OGR1 inhibitor-treated groups. Results are presented as mean \pm SEM. Statistical analysis was performed using one-way ANOVA followed by the post hoc Tukey's test. Water/ vehicle (methylcellulose $0.5 \%)(n=7)$, vehicle/DSS $(n=7)$, and OGR1 inhibitor/DSS: 25, 50, and $100 \mathrm{mg} / \mathrm{kg}(n=6,7$, and 7 , respectively). ${ }^{* *} p<0.01 ;{ }^{* * *} p<0.001$. DSS, dextran sulfate sodium; OGR1, ovarian cancer $G$ protein-coupled receptor 1 . 


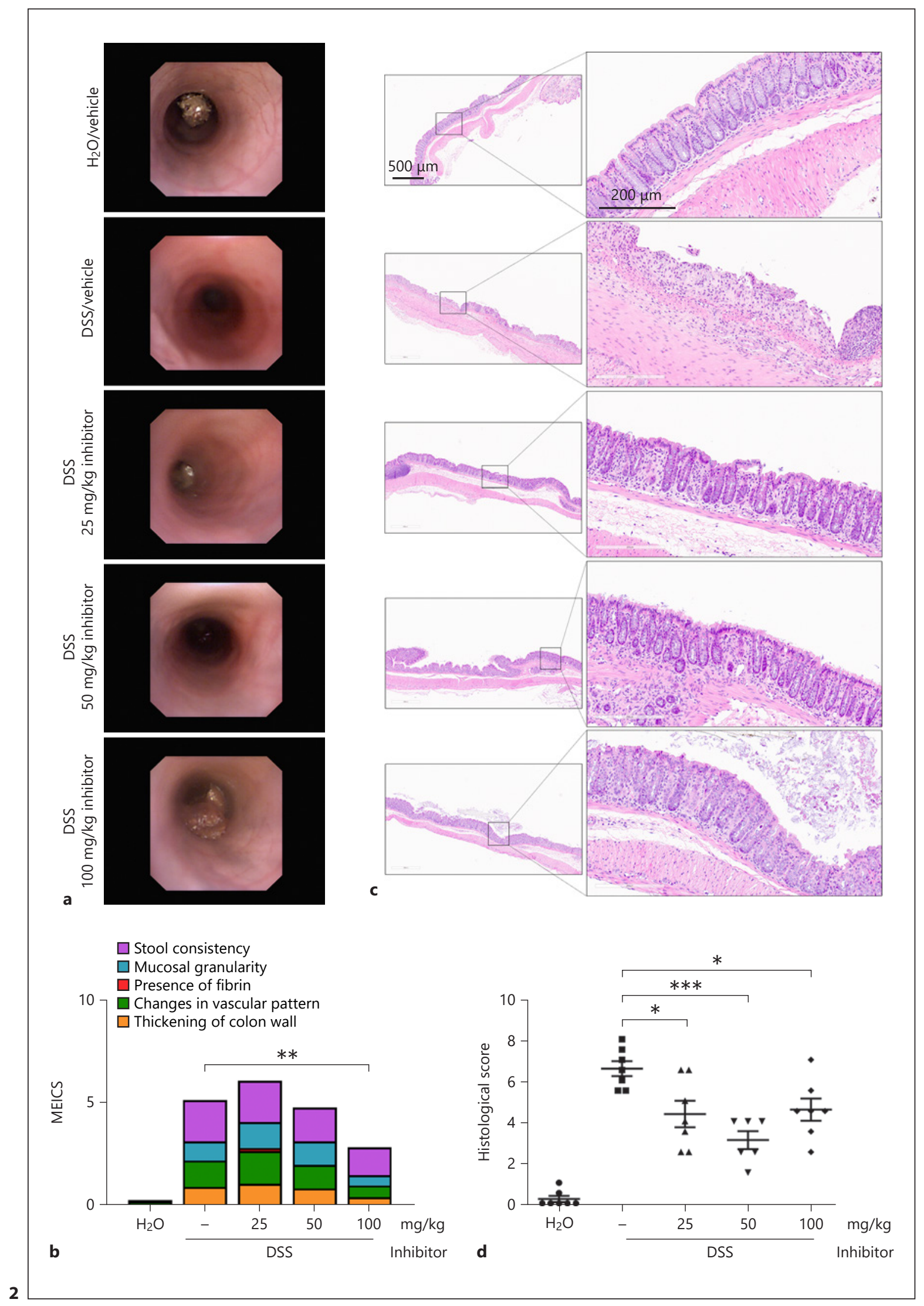

(For legend see next page.) 


\section{Results}

\section{OGR1 Inhibition Has a Mild Protective Effect in}

Acute Dextran Sulfate Sodium (DSS)-Induced Murine Colitis

To study the anti-inflammatory properties of the novel small-molecule OGR1 inhibitor, C57BL/6 mice were given vehicle or OGR1 inhibitor twice daily (b.i.d. 25, 50, and $100 \mathrm{mg} / \mathrm{kg}$ ) by oral gavage during the course of the acute colitis model (Fig. 1a). Acute DSS colitis, induced by the addition of 1.5\% DSS in drinking water for 7 days (Fig. 1a), results in a well-established sequence of histopathological events, such as crypt distortion, shortening, and dropout, occurring in parallel with an inflammatory response [34, 35]. Vehicle-DSS-treated mice developed colitis, as evident from the body weight loss (Fig. 1b), decreased colon length (Fig. 1c, d), increased spleen weight (Fig. 1e, f), colonoscopy (Fig. 2a), increased MEICS (Fig. 2b), H\&E-stained distal and proximal colonic tissue sections (Fig. 2c), and histological score (Fig. 2d).

\section{Macroscopic Disease Indicators Are Reduced in Mice}

Treated with the OGR1 Inhibitor

No significant differences in body weight were observed when comparing the 3 groups treated with the OGR1 inhibitor to the vehicle-water and DSS groups (Fig. 1b); however, macroscopic disease indicators suggest a protective effect in the inhibitor-treated animals: colon shortening was significantly reduced, indicating an attenuation of colon inflammation (Fig. 1c, d). Moreover, endoscopy revealed similar vascularity in the inhibitortreated groups compared to the vehicle-water control animals, that is, no visible wall thickening or fibrin (Fig. 2a, b). In the OGR1 inhibitor groups, the MEICS decreased in a dose-dependent manner at 25,50 , and $100 \mathrm{mg} / \mathrm{kg}$ inhibitor concentrations (Fig. 2b).

\section{Histological Features Are Reduced in the Colon of Mice Treated with the OGR1 Inhibitor}

The effects of OGR1 inhibition at the histopathological level, assessed by H\&E staining, revealed that inhibi-

Fig. 2. OGR1 inhibitor reduces macroscopic disease indicators of intestinal inflammation in acute DSS-induced colitis. Endoscopy was performed, and images were scored according to the MEICS criteria (a, b). Representative pictures are shown (a). Colon specimens were stained with hematoxylin and eosin (c); epithelial damage and leukocyte infiltration scores were assessed in the distal colon (d). Representative pictures are shown (c). Scale bars, 200 and $500 \mu \mathrm{m}$. Results are presented as mean \pm SEM. Statistical anal- tor-treated mice exhibited markedly less infiltration and epithelial damage in the distal colon compared to vehicleDSS mice (Fig. 2c, d). Proliferating cells were detected by anti-Ki-67 antibody and infiltrating T cells by anti-CD3 antibody. Ki-67-positive cells were slightly increased in inhibitor-treated mice compared to vehicle-DSS mice (Fig. 3a). Moreover, decreased infiltration of CD3-positive $\mathrm{T}$ cells was observed in DSS mice administered with the OGR1 inhibitor when compared to vehicle-DSS mice (Fig. 3b).

\section{OGR1 Inhibition Is Protective in Chronic DSS- \\ Induced Murine Colitis}

The acute DSS model, although relevant in studying the physiology of acute flares, wound healing, and resolution of acute inflammation, provides only limited information, since the chemical injury to the epithelial barrier leads to self-limiting inflammation rather than to chronic disease. Therefore, we next tested the OGR1 inhibitor in the chronic DSS model using C57BL/6 WT mice and Ogr1 ${ }^{-/-}$C57BL/6 mice as negative controls. Since $50 \mathrm{mg} /$ $\mathrm{kg}$ appeared to be the optimal dosage of the 3 doses tested in the acute colitis model $(25,50$, and $100 \mathrm{mg} / \mathrm{kg}$ per day), this was the chosen dosage for our chronic DSS colitis model.

\section{OGR1 Inhibition Reduces Clinical Severity and \\ Macroscopic Disease Indicators of Colitis}

Chronic colitis was induced by repeated cycles of $2 \%$ DSS administered for 7 days to C57BL/6 WT and OGR1 KO mice, followed by a recovery phase of 10 days (Fig. 4a). OGR1 inhibitor-treated mice and OGR1 KO mice were protected from body weight loss after the first DSS cycle. WT vehicle-DSS mice lost $\sim 17 \%$ body weight by day 9 , while OGR1 KO mice and OGR1 inhibitor-treated mice lost $\sim 12 \%$ body weight (Fig. $4 \mathrm{a}$ ). The same trend continued after DSS cycles 2 and 3, albeit with a slight decrease in the protective effect of the inhibitor (Fig. 4a).

In line with the results from the acute colitis model, in the chronic colitis model, we observed a decrease in the macroscopic disease indicators in the OGR1 inhibitorhoc Tukey's test. Water/vehicle (methylcellulose $0.5 \%)(n=7)$, vehicle/DSS $(n=7)$, and OGR1 inhibitor/DSS: 25, 50, and $100 \mathrm{mg} /$ $\mathrm{kg}\left(n=6,7\right.$, and 7 , respectively). ${ }^{*} p<0.05,{ }^{* *} p<0.01$, and ${ }^{* * *} p<$ 0.001 . OGR1, ovarian cancer G protein-coupled receptor 1; MEICS, Murine Endoscopic Index of Colitis Severity; DSS, dextran sulfate sodium. 


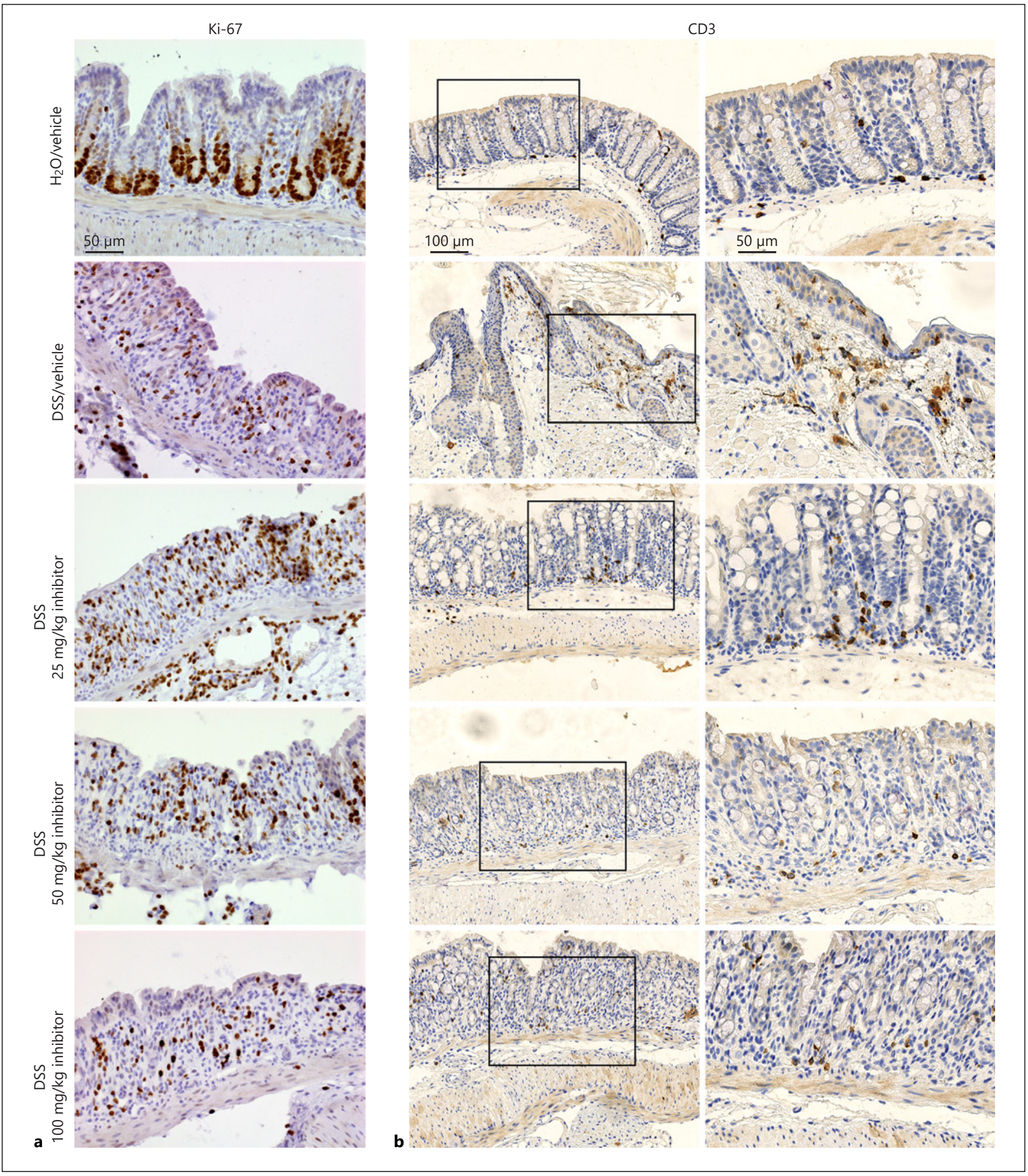

Fig. 3. OGR1 inhibitor reduces infiltration of $\mathrm{T}$ cells and increases cell proliferation in acute DSS-induced colitis. Cell proliferation was assessed in DSS-treated mice administered with the OGR1 inhibitor by immunostaining for Ki-67 (a). Infiltration of T cells (b) was assessed by immunostaining for CD3. Scale bars, 50 and 100 $\mu \mathrm{m}$. OGR1, ovarian cancer G protein-coupled receptor 1; DSS, dextran sulfate sodium. 


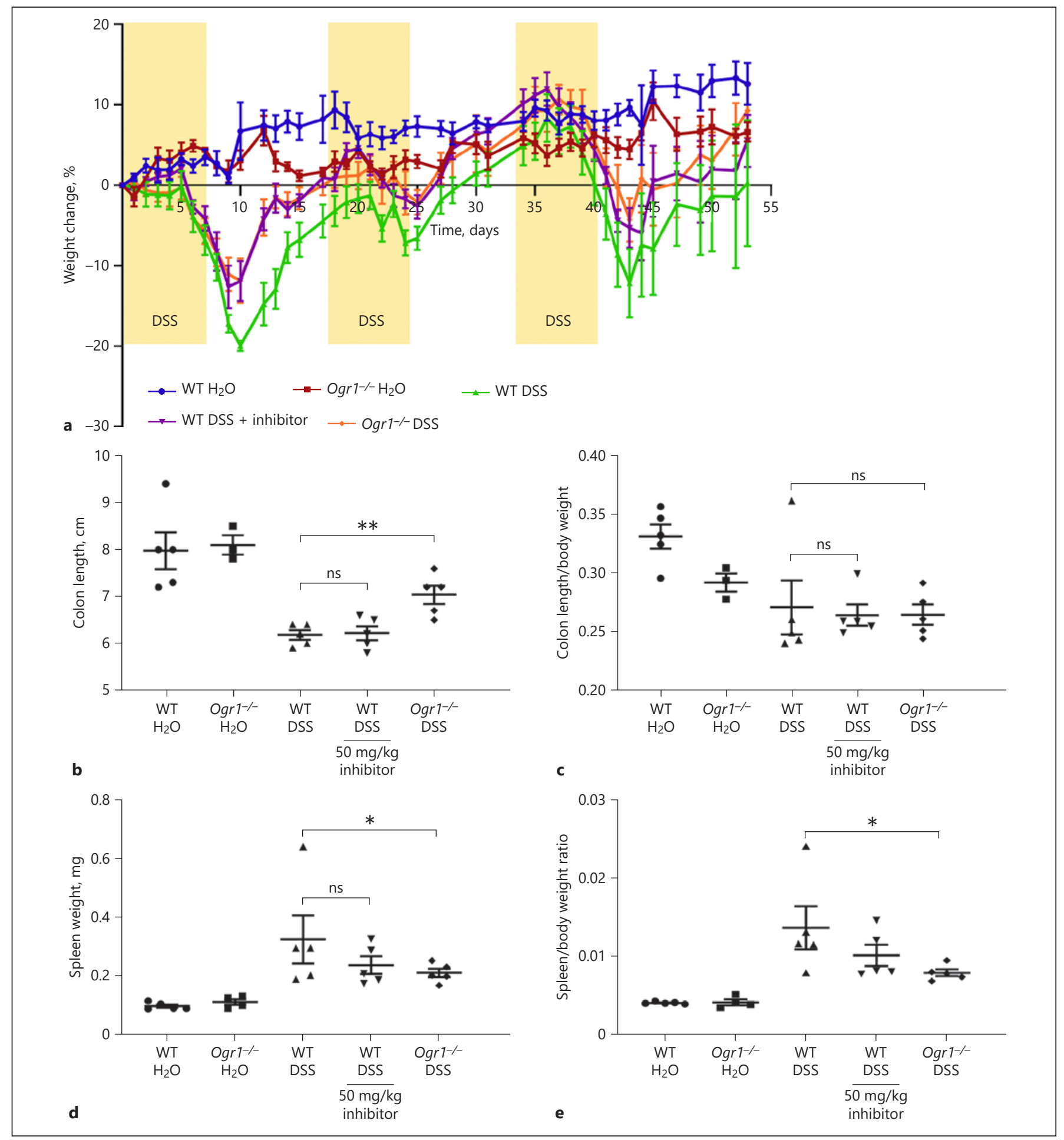

Fig. 4. OGR1 inhibitor reduces clinical severity in chronic DSS-induced colitis. Chronic colitis was induced with 3 cycles of DSS for 7 days followed by drinking water for 10 days in WT and $\mathrm{Ogrl}^{-/-}$mice. The OGR1 inhibitor was administered in the water recovery period by oral gavage twice daily (b.i.d.) at $50 \mathrm{mg} / \mathrm{kg}$ day. Body weight changes (a) were monitored daily and expressed as relative change of body weight in $\%$ relative to day 0 . Mouse colon length $(\mathbf{b}, \mathbf{c})$ and

splenic enlargement $(\mathbf{d}, \mathbf{e})$ were also assessed upon tissue collection on day 55 . Data are presented as mean \pm SEM, and statistical analysis was performed using the $t$-test comparing vehicle- and OGR1 inhibitor-treated groups. WT/water $(n=5), \operatorname{Ogr} 1^{-1-} /$ water $(n=4), \mathrm{WT} /$ DSS $(n=5)$, WT/DSS + OGR1 inhibitor $(n=5)$, and Ogr1 ${ }^{-1} /$ DSS $(n=5) .{ }^{*} p<0.05 ;{ }^{* *} p<0.01$. OGR1, ovarian cancer G protein-coupled receptor 1; DSS, dextran sulfate sodium; WT, wild type. 

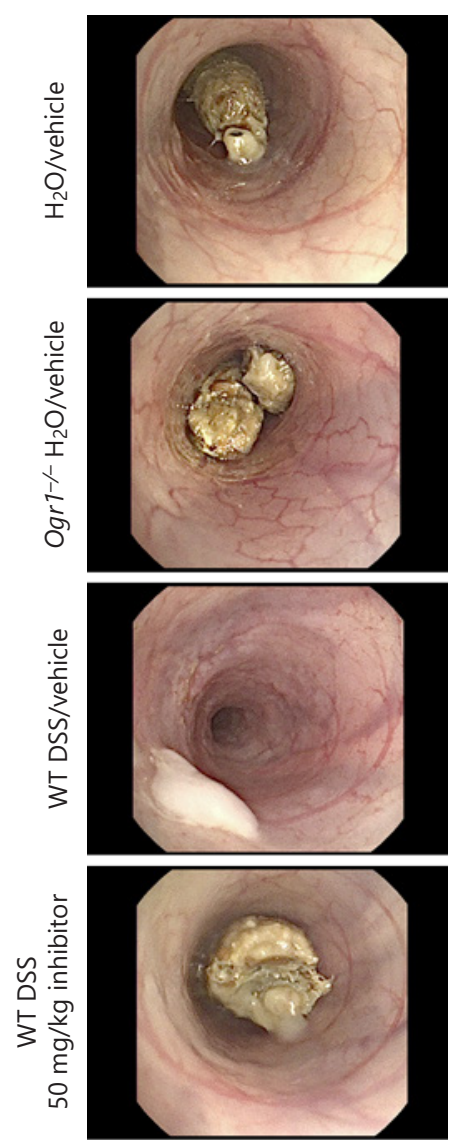

a

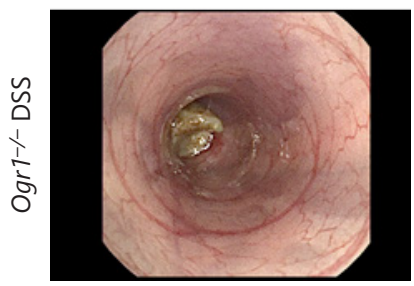

b
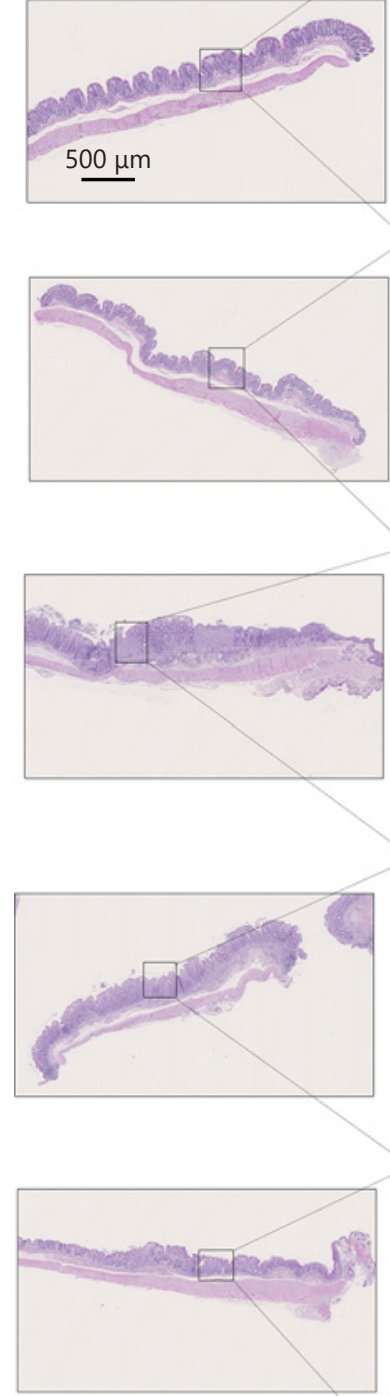
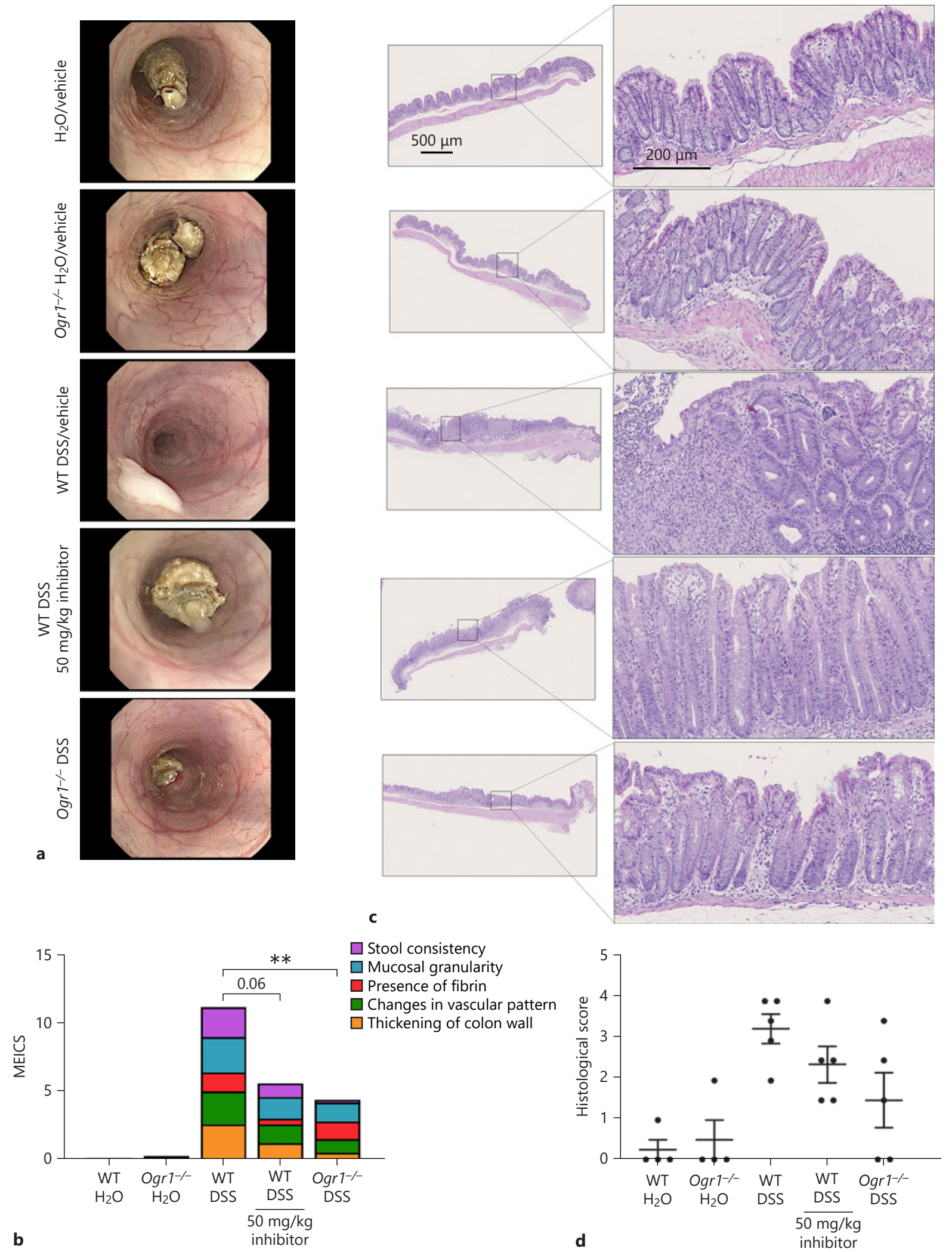

5

(For legend see next page.) 
treated group compared to the WT vehicle-DSS group. Colon shortening in the inhibitor-treated mice was slightly reduced, although not significantly, while the OGR1 KO-DSS group exhibited a significant reduction in colon shortening compared to the WT vehicle-DSS group (Fig. 4b, c). We observed a trend toward decreased spleen weight to body weight ratio between the inhibitor-treated mice and the WT vehicle-DSS mice (Fig. 4d, e), suggesting a mild reduction in disease severity. Moreover, the OGR1 KO-DSS group displayed significantly reduced splenic expansion compared to the WT vehicle-DSS group (Fig. 4d, e).

Endoscopic examination of the OGR1 inhibitor-treated mice revealed no visible fibrin, a marked reduction in wall thickening, and granularity, with similar vascularity and transparency compared to the water control group; however, a looser stool consistency was evident (Fig. 5a). The MEICS of the OGR1 inhibitor-treated mice was markedly reduced compared to the WT vehicle-DSS group and only slightly higher than the OGR1 KO-DSS group (Fig. 5b).

Colonic inflammation was also examined by histology (Fig. 5c); WT vehicle-DSS mice showed severe lesions throughout the mucosa, alteration of the epithelial structure, mononuclear leukocyte infiltration into the mucosal and submucosal areas, and loss of crypts, whereas the inhibitor-treated mice exhibited mild distortion of the crypt structure, less infiltration of mononuclear cells, and mucosal and epithelial damage (Fig. 5c, d).

\section{OGR1 Inhibition Reduces Pro-Inflammatory \\ Parameters of Colitis}

In order to characterize the macrophage infiltration in the colon, IHC staining for the macrophage phenotypic markers F4/80 was performed. This revealed a significant reduction of infiltrating macrophages in the OGR1 inhibitor-treated group compared to the WT vehicle-DSS group (Fig. 6a, b). In addition, mRNA expression of the pro-inflammatory markers TNF (Fig. 6c) and IL-6 (Fig. 6d) in colonic tissue of the OGR1 inhibitor-treated group displayed a trend toward a decrease compared to the WT vehicle-DSS group. Similarly, a slight decrease in

Fig. 5. OGR1 inhibitor reduces macroscopic disease indicators of intestinal inflammation in chronic DSS-induced colitis. Endoscopy was performed, and images were scored according to the MEICS criteria (a, b). Representative pictures are shown (a). Colon specimens were stained with hematoxylin and eosin (c); epithelial damage and leukocyte infiltration scores were assessed in the distal part of the colon (d). Representative pictures are shown (c). Scale
mRNA expression of the fibrosis marker TGF- $\beta 1$ (Fig. 6e) in the OGR1 inhibitor-treated group compared to the WT vehicle-DSS group was observed.

\section{Discussion}

We have previously demonstrated that activation of OGR1 by acidosis ( $\mathrm{pH}$ 6.8) increases the expression of inflammation and immune response, actin cytoskeleton, and cell adhesion genes in intestinal macrophages [24]. Additionally, in an intestinal epithelial cell model, acidic activation of OGR1 increased the expression of genes related to cytoskeleton remodeling, cell adhesion, and growth factor signaling [9]. Furthermore, we recently showed that hypoxia, known to cross-talk with the NF- $\kappa \mathrm{B}$ pathway, enhanced TNF-mediated OGR1 expression, which was reversed in the presence of NF- $\kappa B$ inhibitors [25]. We have also observed a protective effect of OGR1 deficiency in the $I L-10^{-/-}$murine model of spontaneous colitis [24]. In a recent study, we could show that sensing extracellular $\mathrm{pH}$ by OGR1 triggers endoplasmic reticulum stress through the IRE1a-JNK signaling pathway, as well as late-stage autophagy [27], two processes that have been linked to inflammation $[36,37]$. Overall, our previous studies have evidenced a key role of proton-sensing OGR1 in the modulation of intestinal inflammation, but no efforts have been made to investigate the effects of pharmacological modulators of OGR1 in intestinal inflammation. Therefore, in this study, we set to evaluate the effect of a novel small-molecule inhibitor of OGR1 in DSS-induced colitis mouse models. Our results show that this novel OGR1 antagonist has an ameliorating effect on the extent of DSS-induced acute and chronic murine colitis, in particular reducing macroscopic disease indicators and pro-inflammatory indicators of colitis (i.e., including infiltrating $\mathrm{T}$ cells and macrophages) and showing a trend toward a reduction in the expression of the pro-inflammatory cytokines TNF and IL- 6 and the fibrosis marker TGF- $\beta 1$, although the OGR1 inhibitor had an overall lower impact in chronic colitis when compared to acute colitis. Of note, we did not observe a dose-dependent in-

bars, 200 and $500 \mu \mathrm{m}$. Data are presented as mean \pm SEM, and statistical analysis was performed using the $t$-test comparing vehicleand OGR1 inhibitor-treated groups. WT/water $(n=5), \operatorname{Ogr}^{-/-}$/ water $(n=4), \mathrm{WT} / \mathrm{DSS}(n=5), \mathrm{WT} / \mathrm{DSS}+$ OGR1 inhibitor $(n=$ $5)$, and OGR $1^{-1-} / \mathrm{DSS}(n=5) .{ }^{* *} p<0.01$. OGR1, ovarian cancer G protein-coupled receptor 1; MEICS, Murine Endoscopic Index of Colitis Severity; DSS, dextran sulfate sodium; WT, wild type. 


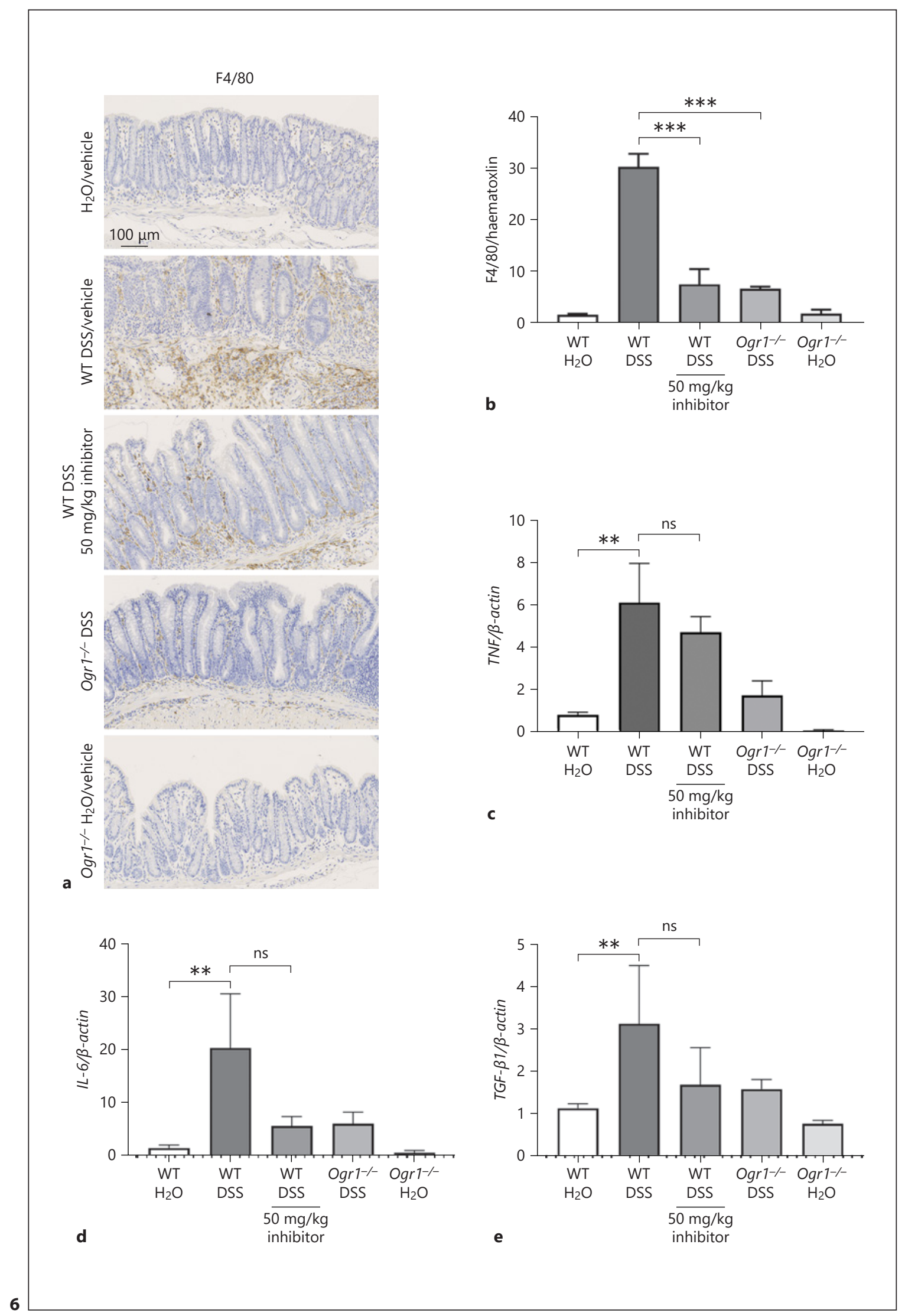

(For legend see next page.) 
hibitory effect in the presence of the OGR1 inhibitor, indicating that the concentration range used in this study was not suitable to observe a dose effect. Further studies using a different concentration range are warranted.

Currently, the therapeutic options for IBD include 5-aminosalicylates, corticosteroids, thiopurines, methotrexate, and calcineurin inhibitors. In addition, there are three classes of biologicals available to treat IBD, including anti-TNF antibodies, antagonists to integrins, and anti-IL-12/IL-23 therapies. Unfortunately, a subset of at least $20 \%$ of patients do not respond to established treatments, and a similar proportion of patients become refractory due to a loss of response [38]. Consequently, there is a pressing need for new therapeutic agents to significantly ameliorate IBD in therapy-refractory patients. In recent years, there has been a resurgence of interest in small molecules for the treatment of IBD. Currently, JAK inhibitors and sphingosine-1-phosphate receptor modulators are being tested in clinical trials [39]. As different signaling cascades play a role in the development of IBD in different patients, a range of drugs targeting different pathways may be required to treat this complex disease. Our results suggest that OGR1 could also constitute a potential target for small-molecule inhibitors for the treatment of IBD.

Due to a defined amount of the available inhibitor, we were required to limit the number of animals in the inhibitor treatment groups and also in the number of DSS cycles in our chronic model, that is, 3 DSS cycles and 5 animals per group. We previously observed that OGR1 deficiency is associated with a decrease in the development of fibrosis in intestinal inflammation [26], but due to these constrictions, this particular model was unsuitable to study the effect of the OGR1 inhibitor on the development of fibrosis in intestinal inflammation. We observed a decrease in TGF- $\beta 1$ mRNA expression in the DSS OGR1 inhibitor-treated group compared to the WTDSS group. However, these differences were not significant. The limited number of animals used in this study invites caution in driving conclusions, and further studies with a minimum of 8 animals per condition and a fourth DSS cycle in the chronic colitis model are warranted.

Fig. 6. OGR1 inhibitor reduces macrophage infiltration in chronic DSS-induced colitis. Infiltration of macrophages was assessed in DSS-treated WT and $\mathrm{Ogrl}^{-/-}$mice by immunostaining for F4/80. Scale bar, $100 \mu \mathrm{m}(\mathbf{a})$. Quantification was performed using ImageJ (b). Data are presented as mean $\pm \mathrm{SEM}$, and statistical analysis was performed using one-way ANOVA; number of pictures per group is $\geq 60$. TNF (c), IL-6 (d), and TGF- $\beta 1$ (e) mRNA expression levels

OGR1 Antagonist Attenuates Intestinal Inflammation
Recently, pH-sensing GPR4, which is predominately expressed in vascular endothelial cells, has also been shown to be a potential therapeutic target for intestinal inflammation [40]. Sanderlin and coworkers [40] demonstrated that a GPR4 inhibitor, previously reported to reduce inflammation in an arthritis model and angiogenesis in a mouse implant model [41], provides a protective effect in the DSS-induced acute colitis mouse model [40]. These authors observed amelioration in disease severity and intestinal histopathology, reduced leukocyte infiltration, and a decrease in the expression of inflammatory and endothelial cell adhesion genes [40]. Our data do not support a redundancy between OGR1 and GPR4, since GPR4 does not appear to compensate for the loss of OGR1 signaling in the presence of the OGR1 inhibitor. Nonetheless, it is plausible that the concomitant use of GRP4 and OGR1 inhibitors would further decrease intestinal inflammation in our models. In line with this, GPR4 is highly expressed in endothelial cells, whereas OGR1, although considered to be ubiquitous, is highly expressed in myeloid and lymphoid cells. On the other hand, another proton-sensing GPCR, TDAG8, which is highly expressed in immune cells is known to play an anti-inflammatory role. Moreover, TDAG8 expression is increased in DSS colitis [28]. Nonetheless, we have not addressed TDAG8 or GPR4 expression or function in OGR1 KO mice, and lack of OGR1 activation in the presence of the OGR1 inhibitor can account for the anti-inflammatory effect of OGR1. Further research is needed to elucidate the individual contribution of these proton-sensing receptors and their mutual regulation in experimental colitis.

To our knowledge, the present study is the first to assess the impact of an OGR1 inhibitor on experimental colitis. Our results demonstrate that the administration of an OGR1 inhibitor reduces macroscopic disease severity and histopathological features in the acute and chronic DSS colitis murine models. The synthesis of small-molecule inhibitors for $\mathrm{pH}$-sensing receptors appears to be a therapeutic opportunity for IBD. Ongoing studies on the function of these $\mathrm{pH}$ receptors could not only improve our understanding of the pathogenesis of IBD but also provide novel therapeutic targets for the treatment of IBD.

normalized to $\beta$-actin of tissue from terminal colon sections. Data are presented as mean \pm SEM, and statistical analysis was performed using one-way ANOVA. WT/water $(n=5)$, Ogr1 ${ }^{-/-} /$water $(n=4), \mathrm{WT} / \mathrm{DSS}(n=5), \mathrm{WT} / \mathrm{DSS}+$ OGR1 inhibitor $(n=5)$, and Ogr $1^{-1-} / \mathrm{DSS}(n=5) .{ }^{* *} p<0.01 ;{ }^{* * *} p<0.001$. OGR1, ovarian cancer $\mathrm{G}$ protein-coupled receptor 1; DSS, dextran sulfate sodium; WT, wild type. 


\section{Statement of Ethics}

All animal experiments were performed according to Swiss animal welfare laws and were approved by the Veterinary Office of the Canton Zurich, Switzerland (Registration No. ZH242/2016).

\section{Conflict of Interest Statement}

G.R. discloses grant support from AbbVie, Ardeypharm, MSD, FALK, Flamentera, Novartis, Roche, Tillots, UCB, and Zeller. M.H. discloses grant support from AbbVie and Novartis. K.B., P.B., Ma.S., C.M., C.S., K.A., S.L., M.R.S., Mi.S., C.V., and P.A.R-C. have no conflicts of interest to disclose.

\section{Funding Sources}

This work was supported by research grants from the Swiss National Science Foundation: Grant No. 310030_172870 and 314730_153380 to G.R. and Grant No. 314730_166381 and 320030_184753 to Mi.S. and from the Stiftung Experimentelle Bio- medizin to Mi.S. The funding institutions had no role in the study design; in the collection, analysis, and interpretation of data; or in the writing of the manuscript.

\section{Author Contributions}

K.B., P.B., Ma.S., C.M., C.S., K.A,. M.R.S., C.V., and M.H. performed experiments; G.R., M.H., Mi.S., and C.V. planned experiments; K.B., M.R.S., C.V., and M.H. analyzed the data; K.B., C.V., P.A.R.C., M.H., and G.R. wrote the manuscript; S.L. and Mi.S. performed administrative and technical support and critical revision of the manuscript for important intellectual content; G.R. and Mi.S. obtained funding for the project; all authors read and approved the manuscript.

\section{Data Availability Statement}

The data underlying this article will be shared on reasonable request to the corresponding author.

\section{References}

1 Raimundo AH, Evans DF, Rogers J, Silk DBA. Gastrointestinal $\mathrm{pH}$ profiles in ulcerative colitis. Gastroenterology. 1992;102:A 681.

2 Fallingborg J, Christensen LA, Jacobsen BA, Rasmussen SN. Very low intraluminal colonic $\mathrm{pH}$ in patients with active ulcerative colitis. Dig Dis Sci. 1993;38(11):1989-93.

3 Nugent SG, Kumar D, Rampton DS, Evans DF. Intestinal luminal $\mathrm{pH}$ in inflammatory bowel disease: possible determinants and implications for therapy with aminosalicylates and other drugs. Gut. 2001;48(4):571-7.

4 Ludwig MG, Vanek M, Guerini D, Gasser JA, Jones CE, Junker U, et al. Proton-sensing Gprotein-coupled receptors. Nature. 2003; 425(6953):93-8.

5 Ishii S, Kihara Y, Shimizu T. Identification of T cell death-associated gene 8 (TDAG8) as a novel acid sensing G-protein-coupled receptor. J Biol Chem. 2005;280(10):9083-7.

6 Seuwen K, Ludwig MG, Wolf RM. Receptors for protons or lipid messengers or both. J Recept Signal Transduct Res. 2006;26(5-6):599610.

7 Tomura H, Wang JQ, Komachi M, Damirin A, Mogi C, Tobo M, et al. Prostaglandin I(2) production and cAMP accumulation in response to acidic extracellular $\mathrm{pH}$ through OGR1 in human aortic smooth muscle cells. J Biol Chem. 2005;280(41):34458-64.

8 Wang J, Sun Y, Tomura H, Okajima F. Ovarian cancer G-protein-coupled receptor 1 induces the expression of the pain mediator prostaglandin E2 in response to an acidic extracellular environment in human osteoblastlike cells. Int J Biochem Cell Biol. 2012;44(11): 1937-41.
9 de Valliere C, Vidal S, Clay I, Jurisic G, Tcymbarevich I, Lang $\mathrm{S}$, et al. The $\mathrm{pH}$-sensing receptor OGR1 improves barrier function of epithelial cells and inhibits migration in an acidic environment. Am J Physiol Gastrointest Liver Physiol. 2015;309(6):G475-90.

10 Wei WC, Bianchi F, Wang YK, Tang MJ, Ye $\mathrm{H}$, Glitsch MD. Coincidence detection of membrane stretch and extracellular $\mathrm{pH}$ by the proton-sensing receptor OGR1 (GPR68). Curr Biol. 2018;28(23):3815-23.e4.

11 Ray K. IBD: the changing epidemiology of IBD. Nat Rev Gastroenterol Hepatol. 2017; 14(12):690

12 Lowenberg M, D’Haens G. Next-generation therapeutics for IBD. Curr Gastroenterol Rep. 2015; 17(6):21.

13 Fiocchi C. Inflammatory bowel disease: etiology and pathogenesis. Gastroenterology. 1998;115(1):182-205.

14 Podolsky DK. Inflammatory bowel disease. N Engl J Med. 2002;347(6):417-29.

15 Kaser A, Zeissig S, Blumberg RS. Inflammatory bowel disease. Annu Rev Immunol. 2010; 28:573-621.

16 Rieder F, Brenmoehl J, Leeb S, Schölmerich J, Rogler G. Wound healing and fibrosis in intestinal disease. Gut. 2007;56(1):130-9.

17 Silva FA, Rodrigues BL, Ayrizono ML, Leal RF. The immunological basis of inflammatory bowel disease. Gastroenterol Res Pract. 2016;2016:2097274.

18 Press AG, Hauptmann IA, Hauptmann L, Fuchs B, Fuchs M, Ewe K, et al. Gastrointestinal $\mathrm{pH}$ profiles in patients with inflammatory bowel disease. Aliment Pharmacol Ther. 1998;12(7):673-8.
19 Lardner A. The effects of extracellular $\mathrm{pH}$ on immune function. J Leukoc Biol. 2001;69(4): 522-30.

20 Tannahill GM, O'Neill LA. The emerging role of metabolic regulation in the functioning of Toll-like receptors and the NOD-like receptor Nlrp3. FEBS Lett. 2011;585(11):1568-72.

21 Krawczyk CM, Holowka T, Sun J, Blagih J, Amiel E, DeBerardinis RJ, et al. Toll-like receptor-induced changes in glycolytic metabolism regulate dendritic cell activation. Blood. 2010;115(23):4742-9.

22 Roiniotis J, Dinh H, Masendycz P, Turner A, Elsegood CL, Scholz GM, et al. Hypoxia prolongs monocyte/macrophage survival and enhanced glycolysis is associated with their maturation under aerobic conditions. I Immunol. 2009;182(12):7974-81.

23 Menkin V. Biology of inflammation; chemical mediators and cellular injury. Science. 1956;123(3196):527-34.

24 de Valliere C, Wang Y, Eloranta JJ, Vidal S, Clay I, Spalinger MR, et al. Protein-coupled $\mathrm{pH}$-sensing receptor OGR1 is a regulator of intestinal inflammation. Inflamm Bowel Dis. 2015;21(6):1269-81.

25 de Valliere C, Cosin-Roger J, Simmen S, Atrott K, Melhem H, Zeitz J, et al. Hypoxia positively regulates the expression of $\mathrm{pH}$ sensing G-protein-coupled receptor OGR1 (GPR68). Cell Mol Gastroenterol Hepatol. 2016;2(6):796-810.

26 Hutter S, van Haaften WT, Hünerwadel A, Baebler K, Herfarth N, Raselli T, et al. Intestinal activation of $\mathrm{pH}$-sensing receptor OGR1 [GPR68] contributes to fibrogenesis. J Crohns Colitis. 2018;12(11):1348-58. 
27 Maeyashiki C, Melhem H, Hering L, Baebler $\mathrm{K}$, Cosin-Roger J, Schefer F, et al. Activation of $\mathrm{pH}$-sensing receptor OGR1 (GPR68) induces ER stress via the IRE1alpha/JNK pathway in an intestinal epithelial cell model. Sci Rep. 2020;10(1):1438.

28 Tcymbarevich I, Richards SM, Russo G, Kuhn-Georgijevic J, Cosin-Roger J, Baebler $\mathrm{K}$, et al. Lack of the $\mathrm{pH}$-sensing receptor TDAG8 [GPR65] in macrophages plays a detrimental role in murine models of inflammatory bowel disease. J Crohns Colitis. 2019; 13(2):245-58.

29 Tcymbarevich IV, Elegant JJ, Russell JB, Obialo N, Spalinger M, Cosin-Roger J, et al. The impact of the rs8005161 polymorphism on G protein-coupled receptor GPR65 (TDAG8) $\mathrm{pH}$-associated activation in intestinal inflammation. BMC Gastroenterol. 2019;19(1):2.

30 Wang $\mathrm{Y}$, de Vallière $\mathrm{C}$, Imenez Silva $\mathrm{PH}$ Leonardi I, Gruber S, Gerstgrasser A, et al. The proton-activated receptor GPR4 modulates intestinal inflammation. J Crohns Colitis. 2018;12(3):355-68.
31 Mohebbi N, Benabbas C, Vidal S, Daryadel A, Bourgeois S, Velic A, et al. The proton-activated $G$ protein coupled receptor OGR1 acutely regulates the activity of epithelial proton transport proteins. Cell Physiol Biochem. 2012;29(3-4):313-24.

32 Fischbeck A, Leucht K, Frey-Wagner I, Bentz S, Pesch T, Kellermeier S, et al. Sphingomyelin induces cathepsin D-mediated apoptosis in intestinal epithelial cells and increases inflammation in DSS colitis. Gut. 2011;60(1):55-65.

33 Hausmann M, Obermeier F, Paper DH, Balan $\mathrm{K}$, Dunger N, Menzel K, et al. In vivo treatment with the herbal phenylethanoid acteoside ameliorates intestinal inflammation in dextran sulphate sodium-induced colitis. Clin Exp Immunol. 2007;148(2):373-81.

34 Cooper HS, Murthy SN, Shah RS, Sedergran DJ. Clinicopathologic study of dextran sulfate sodium experimental murine colitis. Lab Invest. 1993;69(2):238-49.

35 Okayasu I, Hatakeyama S, Yamada M, Ohkusa T, Inagaki Y, Nakaya R. A novel method in the induction of reliable experimental acute and chronic ulcerative colitis in mice. Gastroenterology. 1990;98(3):694-702.

36 Cao SS. Endoplasmic reticulum stress and unfolded protein response in inflammatory bowel disease. Inflamm Bowel Dis. 2015; 21(3):636-44.
37 Cosin-Roger J, Simmen S, Melhem H, Atrott K, Frey-Wagner I, Hausmann M, et al. Hypoxia ameliorates intestinal inflammation through NLRP3/mTOR downregulation and autophagy activation. Nat Commun. 2017; 8(1):98.

38 Wong U, Cross RK. Primary and secondary nonresponse to infliximab: mechanisms and countermeasures. Expert Opin Drug Metab Toxicol. 2017;13(10):1039-46.

39 Sabino J, Verstockt B, Vermeire S, Ferrante $\mathrm{M}$. New biologics and small molecules in inflammatory bowel disease: an update. Therap Adv Gastroenterol. 2019; 12 1756284819853208.

40 Sanderlin EJ, Marie M, Velcicky J, Loetscher $\mathrm{P}$, Yang LV. Pharmacological inhibition of GPR4 remediates intestinal inflammation in a mouse colitis model. Eur J Pharmacol. 2019; 852:218-30.

41 Velcicky J, Miltz W, Oberhauser B, Orain D, Vaupel A, Weigand K, et al. Development of selective, orally active GPR4 antagonists with modulatory effects on nociception, inflammation, and angiogenesis. J Med Chem. 2017; 60(9):3672-83. 